INPLASY

PROTOCOL

To cite: Bao et al. Efficacy and safety of acupuncture combined with Chinese herbal medicine in the treatment of type 2 diabetes mellitus: A protocol for a systematic review and meta-analysis. Inplasy protocol 2021100015. doi: 10.37766/inplasy2021.10.0015

Received: 05 October 2021

Published: 05 October 2021

Corresponding author: Zheng Nan

nanzheng001@aliyun.com

Author Affiliation:

Changchun University of

Chinese Medicine.

Support: None.

Review Stage at time of this submission: The review has not yet started.

Conflicts of interest: None declared.

\section{Efficacy and safety of acupuncture combined with Chinese herbal medicine in the treatment of type 2 diabetes mellitus: A protocol for a systematic review and meta-analysis}

Bao, PJ1; Mi, J2; Yu, ZY3; Liu, L4; Zhu, ZY5; Liu, SL6; Nan, Z7.

Review question / Objective: The aim of this systematic review is to compare acupuncture combined with Chinese herbal medicine and placebo/hypoglycemic drugs in terms of efficacy and acceptability in the T2DM to better inform clinical practice. To this end, the proposed systematic review will address the following question: Which is the best choice to reduce fasting blood glucose, 2-hour postprandial blood glucose (2hG), and glycosylated hemoglobin (HbA1c) in T2DM patients, acupuncture combined with Chinese herbal medicine or placebo/hypoglycemic drugs?

Condition being studied: Diabetes has become a global public health problem and danger to human health. Diabetes is the main cause of blindness, kidney failure, heart attack, stroke and lower limb amputation.According to the latest epidemiological survey and research, the overall prevalence of diabetes in mainland China is $11.2 \%$, of which type 2 diabetes mellitus(T2DM) accounts for more than 90\%.acupuncture combined with Chinese herbal medicine have been widely used in the treatment of T2DM.However, we have not found a meta-analysis of their synergistic effects.Therefore, this systematic review and meta-analysis aims to evaluate the efficacy and safety of acupuncture combined with Chinese herbal medicine in the treatment of T2DM.

INPLASY registration number: This protocol was registered with the International Platform of Registered Systematic Review and Meta-Analysis Protocols (INPLASY) on 05 October 2021 and was last updated on 05 October 2021 (registration number INPLASY2021100015).

\section{INTRODUCTION}

Review question / Objective: The aim of this systematic review is to compare acupuncture combined with Chinese herbal medicine and placebo/hypoglycemic drugs in terms of efficacy and acceptability in the T2DM to better inform clinical practice. To this end, the proposed systematic review will address the following question: Which 
is the best choice to reduce fasting blood glucose, 2-hour postprandial blood glucose (2hG), and glycosylated hemoglobin (HbA1c) in T2DM patients, acupuncture combined with Chinese herbal medicine or placebo/hypoglycemic drugs?

Condition being studied: Diabetes has become a global public health problem and danger to human health. Diabetes is the main cause of blindness, kidney failure, heart attack, stroke and lower limb amputation.According to the latest epidemiological survey and research, the overall prevalence of diabetes in mainland China is $11.2 \%$, of which type 2 diabetes mellitus(T2DM) accounts for more than 90\%.acupuncture combined with Chinese herbal medicine have been widely used in the treatment of T2DM.However, we have not found a meta-analysis of their synergistic effects. Therefore, this systematic review and meta-analysis aims to evaluate the efficacy and safety of acupuncture combined with Chinese herbal medicine in the treatment of T2DM.

\section{METHODS}

Participant or population: Patients enrolled in studies of T2DM screening will be eligible for this review, with no exclusions based on ethnicity or age.

Intervention: Acupuncture combined with Chinese herbal medicine.

Comparator: Placebo/hypoglycemicdrugs.

Study designs to be included: Randomized controlled study.

Eligibility criteria: The inclusion criteria for the literature search will be as follows: (1) studies including patients aged $\geq 18$ years, regardless of sex, with T2DM diagnosed according to the standards of the American Diabetes Association or the World Health Organization; (2) randomized controlled studies (RCTs); (3) studies in which the experimental group was treated with acupuncture combined with Chinese herbal medicine; (4) studies in which the control group was treated with placebo or hypoglycemic drugs. The exclusion criteria will be as follows: (1) studies including patients aged <18, with non-T2DM; (2) studies in which the experimental group was given treatment methods other than acupuncture combined with Chinese herbal medicine; (3) studies in which the control group was not treated with a placebo or a hypoglycemic drug; (4) studies that were not randomized controlled trials (RCTs).

Information sources: The PubMed, Web of Science, Embase, AMED, Cochrane Library, CNKI, VIP, CBM, and Wanfang databases will be searched. The publication date or language will not be limited. We will also check other ongoing and unpublished studies in the clinical trial registry. At the same time, we will manually search all reference lists from relevant systematic reviews to find other eligible studies.

Main outcome(s): The primary outcome measures will include fasting blood glucose, 2-hour postprandial blood glucose (2hG), and glycosylated hemoglobin (HbA1c).

Additional outcome(s): The secondary outcome measures will include triglycerides (TGs), cholesterol (TC), and insulin secretion (INS).

Quality assessment / Risk of bias analysis: The two researchers will use the Cochrane risk bias assessment tool to separately assess the quality of the randomized studies. The assessment content addresses the following: whether the random method is correct; whether the allocation concealment is achieved; whether the blind method is implemented; whether the result data are complete; whether there is selective reporting bias; and whether there are other biases. We will use Begg's and Egger's tests and set $P<0.1$ as statistically significant, and we will use a funnel chart to assess publication bias. If the evaluation quality of any single study is inconsistent between researchers, the discrepancy will be resolved through consensus between the researchers. 
Strategy of data synthesis: We will use Review Manager software (REVMAN v5.3 Cochrane Collaboration) to meta-analyze the selected literature, and P0.05 and $12<50 \%$, there is homogeneity among the studies, and the fixed-effects model will be used for meta-analysis. If $\mathrm{P} \leq 0.05$ and $12 \geq 50 \%$, there is heterogeneity among the studies. Sensitivity analysis will then be used to analyze the source of heterogeneity. After the influence of clinical heterogeneity is excluded, the randomeffects model will be used for metaanalysis.

Subgroup analysis: We will use subgroup analysis based on different interventions, controls, and outcomes.

Sensitivity analysis: Sensitivity analysis will then be used to analyze the source of heterogeneity.

Country(ies) involved: China.

Keywords: acupuncture, Chinese herbal medicine, type 2 diabetes mellitus, metaanalysis, protocol, safety, systematic review.

Contributions of each author:

Author 1 - Pengjie Bao.

Email: baopengjie111@163.com

Author 2 - Jia Mi.

Author 3 - Ziyang Yu.

Author 4 - Le Liu.

Author 5 - Zhiyue Zhu.

Author 6 - Shilin Liu.

Author 7 - Zheng Nan.

Email: nanzheng001@aliyun.com 